\title{
The bop sealing device for testing of layered water injection well Xinggang Guo
}

\author{
The fifth production plant of DaQing oilfield co., LTD of China Petroleum Corporation. DaQing \\ HeiLongjiang. 163513
}

\section{Keywords: Layered testing; sealing device; cable folding proof; secondary seal}

\begin{abstract}
The bop sealing device for testing of layered water injection well is the supporting tools for blowout preventer of layered water injection well test. Many problems exists in the traditional sealing device, such as difficulty for down-pass instruments in testing initial stage, cable bending caused by the shaking of spring docking instrument, testing interruption caused by thorn water, gland has only one handle which is not convenient for rotation, need to be integrally replaced after sealing device main body broken, etc. Aiming at these design problem, a new bop sealing device for testing of layered water injection well is invented, and the low test efficiency, easy to stab the water environmental pollution in the process of test, cable loss more single well test cost increasing problems caused by technique defects of traditional device are solved. From the scene of the experimental area, the new spray seal parts structure size design is reasonable, possess the functions of oil lubrication, cable bending, realizing secondary seal, and rapid unloading gland, etc. After measuring by 26 teams application, the test time is saved for 20 minutes for each single team, cable free from wrecked, saving the cost by 5\%, benefiting RMB 570,000 per year. Currently, the new type bop sealing device for testing of layered water injection well is popularized and applied in oilfield for more than 300 sets, the application effect is favorable, effectively improve the test efficiency and has a great application promotion value.

With the increase of oilfield development difficulty, in order to reduce interlayer conflict and effective play to the potential of various types of reservoir, need to quantitative injection for all kinds of oil layers. To strictly control the water injection quantity, layered water injection well test method has experienced a development from the common electromagnetic flowmeter to measure the linkage of layered water injection system. Improving the layered water injection well stratified water allocation and test quality, and achieve good water injection is an important work for oilfield, injection water, controlling water cut rising velocity, slow down production decline, improving the overall efficiency of water flooding development. Bop sealing device is the matching tool of nozzle used in layered water injection well test technology. As the existence of design problems, in use process there is a problem affecting the safety of test efficiency and test. To efficiently complete the injection well testing work, the key is to develop new type of layered water injection well test the bop sealing device, to reduce cable wear cost savings, lowering instrument smoothly to improve test efficiency, convenient operation of the field test, the purpose of safe environmental protection, with high practical and promotional value.
\end{abstract}

\section{Background}

After entering the high water cut stage mining, oil field water injection management work is the key to late stable yield in the development process, how to well water injection, injection water enough, and adjustment for the decision of oilfield development plan to provide first-hand dynamic data, a good job must be done for layered water injection test, to ensure the quality of layering water injection ${ }^{[1]}$. Currently, the layered water injection well of conventional test methods are single wire winch, using electromagnetic flowmeter, by vote to plug, replace the water nozzle diameter to implement layered water debugging, with underground storage type flowmeter in ground playback flow and pressure data. According to the traffic data of playback and injection allocation scheme comparison results, decide whether to adjust again. However, under the condition of the traffic discrepancy, this method need frequent under downhole plug, replacement of water mouth, test, and adjustment work. 
Aiming at the problems of adjustable volume measurement and measuring adjustable difficulty increasing, efficient real-time measuring technology has gradually began to large-scale popularization and application in recent years. The original single wire winch is improved for wire and cable double-drum hoist, to realizing pneumatic switch function, implement different winch. Working principle is the application of downhole flow adjustable instrument measurement and corresponding downhole flow adjustable plug in downhole automatic docking, the ground input instructions, microcomputer control regulation in the plug flow orifice flow area, so as to achieve stratified flow of real-time monitoring, dynamic adjustment, avoid the cast out by changing water mouth plug work, implements the ground direct reading, synchronization complete of visual points injection wells debugging and testing. Promotion for intelligent measuring adjustment technology has realized completing the whole well and each interval flow testing and interval water adjustment work repeatedly within one trip, and simultaneously testing pressure and temperature at the same time.

If using conventional electromagnetic flowmeter testing and efficient intelligent tuning the two test methods, several problems will occur, such as difficulty for down-pass instruments in testing initial stage, cable bending caused by the shaking of spring docking instrument, testing interruption caused by thorn water, gland has only one handle which is not convenient for rotation, need to be integrally replaced after sealing device main body broken, etc. This seriously influences test efficiency, and has ehs risks. Aiming at the existing disadvantages of sealing device for layered water injection well test, a new type of easy to operate, safe sealing device is developed ${ }^{[2]}$, and this is of great significance for improving the efficiency of water injection test and solving the wellhead sealing device in the process of testing water environmental pollution problems.

\section{Conventional sealing device}

Conventional injection well testing seal is $270 \mathrm{~mm}$ length. In the process of layering water injection well testing operation, because of the large packing friction, not well overcome resistance, packing down early testing instrument is difficult, need testers stood on the platform to send cable is about 300 meters, takes about 20 minutes.

The bottom springs of the seal is connected with instruments by rope socket, which is easy to cable due to seal rock can be easily bended, even broken; once meeting a suddenly thorn water during testing, it must be interrupted for there is no emergency sealing mechanism, and this seriously affects the test efficiency ${ }^{[3]}$; the gland has only one handle, so the rotation is not convenient, operating more arduous; once sealing device main body internal is cable wear, need to replace the entire test sealing device, which costs heavy energy.

For the conventional layered water injection well test sealing device, the cable cannot prevent folding, the success rate of emergency sealing is zero, and the design is not reasonable place, which cannot meet the practical requirements of test site operation. The emergence of these problems, affects the safety of test efficiency and test. So it is urgently needed to improve the sealing device comprehensively in order to achieve three following purposes: first is to reduce the cable wear cost savings; second is to improve the testing efficiency; finally, the field test is easy to operate, safe and environmental friendly.

\section{Novel bob sealing device}

The novel high efficient bob sealing device is made by 45 \# steel, consisting of the nut oil cup, top and bottom sub, secondary seal card, gland, copper sets, short tube and pedestal, etc. ${ }^{[4]}$. The following problems are solved:

The first one is that an oil cup is added in the original seal nut, so that the lubrication oil can be injected at any time during the process of testing, to reduce the friction instrument under the well, reduce the cable wear and prolong its life;

Secondly, the optimization design of emergency seal card, seal card using ptfe material, wear resistance, high temperature resistant, not damage the cable, mainly use it when suddenly 
overflowing sewage in the process of testing, achieve the purpose of sealing, avoid interrupting test, improve test efficiency, safety and environmental protection;

Third is that the short pipe is used to connect the lower of the sealing device to the cable head tightly, to prevent damage caused by the cable seal rock;

Fourth is that multiple handles are welded on the gland evenly around the edge, which makes the discharge operation convenient, efficient, and energy saving;

Fifth is that the internal replaceable copper set is designed, and only this part need to be replaced after wear, which can save the costs.

\section{Experiments}

Test experimental management injection wells possess the total number of 805 wells, the number of layered water injection is 727 , the startup well number 665 , layered water injection well test cycle is 4 months, normal needed workload is 1995 well times, comprehensive adjustment scheme workload is 220 well times, interval subdivision workload is 101 well times. Total of measuring the shift of 26, including conventional electromagnetic flowmeter test team 8 , efficient intelligent measurement test team 18. Experimental zone 26 test teams in 2014, application of new type of layered water injection well test the bop sealing device, adjustable 2316 years accumulative test well, the test result is very good, no cable broken phenomenon, effectively protect the test cable, emergency seal using 35 times, good seal, achieved the expected design goal.

Newly developed efficient blowout sealing device, on-site validation results for cable discount rate of $100 \%$, and $100 \%$ success rate of emergency seal. By using this sealing device, secondary seal is realized, the pressure test is kept, and the continual testing is ensured, additionally, employee safety operation, convenient and quick, reduce labor intensity, improve the test efficiency, realize safe environmental protection can also be satisfied.

\section{Benefit Evaluation}

\subsection{Economic benefit}

(1) Processing costs 1,000 yuan per set, 26 teams processing costs for: $26 \times 0.1=\mathrm{RMB} 26,000$ yuan.

(2) Improve the test efficiency and save test time 20 min per day, monthly savings: $(20 \times$ 22) $/ 60=7.3(\mathrm{~h})$.

Measuring 8 wells per team per month, the effective working time calculated on 22 days, effective work time of $6 \mathrm{~h}$ per day. Average single well test time is $(22 \times 6) / 8=16.5(\mathrm{~h})$.

$7.3 / 16.5=0.44$ more Wells can be tested in one month. Single well test benefit is calculated at 3,000 yuan. A single time in economic benefit is: $0.44 * 0.3 * 12=16,000$ yuan

For currently 26 teams, the economic benefit is $26 \times 1.6=416,000$ yuan.

(3) Lengthen the life time of the cable. Originally, 0.3 tray cable is exchanged, after using the new end cap, 0.2 tray cable is exchanged per year, and the cost of each tray cable is 70,000yuan. The cost saving for 26 teams is $0.1 \times 26 \times 7=180,000$ yuan.

Each year economic benefit: $41.6+18-2.6=570,000$ yuan.

\subsection{Social benefit}

Layered water injection well test bop sealing device has simple structure, reasonable size parts design, reliable function, easy operation features. It can reduce the labor intensity of workers, improve the working efficiency, and is assured by injection well testing operation personnel, appropriated to be applied to replace the conventional sealing device.

\section{Conclusion}

For the designed novel efficient bob sealing device, cable discount rate $100 \%$, For the new designed secondary emergency seal card, by tightening the screw oil cup, the cable hydraulic 
pressure can be hold, achieve 100\% success rate of emergency seal. The device is easy operation, safety, and improved the test efficiency, effectively protected the cable. It has been used in oilfield company for 300 sets, which were certainly affirmed, and has a favorable application promotion value.

\section{Reference}

[1] Zhouli, Zhang Libao, Hou Canhong, et. To improve the test seal test success rate of wells. Oil gas field surface engineering [J]. Daqing Oilfield Co., Ltd., 2005, 24 (06): 58 - 59.

[2] Gaoyang. Injection well blowout preventer test plug. Oil gas field surface engineering [J]. Daqing Oilfield Co., Ltd., 2010, 29 (01): 79 - 80.

[3] Yuan Yeqi, He Hankun, Tao Liping, et al. Testing [J] of oil and gas wells, 2004,13 (07): 98 - 97.

[4] Xu Maogong, GUI Dingyi. Tolerance fit and technical measurement [J]. Mechanical Industry Press, 1995. 